\title{
BIBLIOGRAFIA
}

\section{Bibliografía de y Sobre Luis Durand}

\section{OBRAS DE LUIS DURAND}

A. Libros

I. La Chabela (Santiago: "Lectura selecta", I927).

2. Mal de amor (Santiago: "Lectura selecta", 1928).

3. Tierra de pellines (Santiago: Nascimento, 1929), Segunda edición (Idem., I945).

4. Campesinos (Santiago: Nascimento, r929), Segunda edición (Idem., I950).

5. Cielos del stur (Santiago: "Cultura", r933), Segunda edición (Santiago: Zig-Zag, I946).

6. Mercedes Urizar (Santiago: Nascimento, 1934), Segunda edición (Idem., I952).

7. Piedra que rueda (Santiago: Ercilla, I934).

8. El primer bijo (Santiago: "Cultura", 1936).

9. Visión de Sarmiento (Santiago: Nascimento, 1938).

ro. Mi amigo Pidén (Santiago: Nascimento, 1939).

Ir. Presencia de Chile (Santiago: Nascimento, I942).

12. Vino tinto, y otros cuentos (Santiago: Cruz del Sur, 1943).

13. Casa de la infancia (Santiago: Orbe, 1944), Segunda edición (Idem., I958).

14. La nocbe en el camino (Santiago: Zig-Zag, I945),

Segunda edición (Idem., I952),

Tercera edición (Idem., r962). 
15. Alma y cuerpo de Chile (Santiago: Nascimento, I947).

I6. Guauguau y sus amigos (Santiago: Rapa-Nui, I947).

r7. Frontera (Santiago: Nascimento, I949),

Segunda edición (Idem., I95I).

Tercera edición (Idem., r954).

Cuarta edición (Idem., r958).

18. Sietecuentos (Santiago: Nascimento, 1950),

Segunda edición (Idem., I955),

Tercera edición (Idem., I958).

19. Don Arturo (Santiago: Zig-Zag, I952).

20. Gente de mi tiempo (Santiago: Nascimento, 1953).

21. Paisajes y gentes de Cbile (Santiago: Zig-Zag, r953),

Segunda edición (Idem., I962).

22. Un amor [Obra póstuma] (Santiago: Zig-Zag, 1957),

Segunda edición (Idem., r958),

Tercera edición (Idem., x,60).

\section{B. CUENTOS $Y$ ENSAYOS PUBLICADOS EN ATENEA*}

I. * "La caída del roble" [Cambió el título por "Una tragedia montañesa" en Campesinos], XIII, junio de r930, pp. 383-398.

2. * "Vino tinto", XVI, agosto de I93x, pp. 307-324.

3. " "Visión del campesino chileno", XXIII, junio de 1933, pp. 495503.

4. "Algo sobre el cuento y los cuentistas chilenos", XXIV, agosto de r933, pp. 262-275.

5. "La frontera y su interpretación en la literatura chilena", XXIV, septiembre de 1933, pp. 513-532.

6. ""El desierto fecundo", XXXIV, mayo de 1936 , pp. 227-245.

7. "Apreciación del roto", XXXVII, enero de I937, pp. 28-4I.

8. "Evocando el tiempo en que leímos 'Maria'", XXXVIII, abril de I937, pp. 30-42.

9. *"En el andarivel", XXXVIII, junio de I937, pp. 305-324.

Io. *"El jilguero", XL, noviembre de I937, pp. 260-270.

* Los cuentos están marcados con asteriscos (todos aparecieron posteriormente publicados en libros); los esbozos y ensayos que integran Presencia de Chile, con una $\mathrm{p}$ marginal; $\mathrm{y}$ los que figuran en Alma $y$ cuerpo de Cbile, conuna a marginal. 
II. "Visión de Sarmiento: Su inquietud fecunda y creadora", LIII, septiembre de 1937 , pp. 447-47I.

12. *"El triunfo del Cenizo", LIV, noviembre de I938, pp. 230-246.

13. " "Interpretación de la cueca", LVI, mayo de r939, pp. 258-266.

I4. " "Un niño de provincia llega a Santiago", LXIII, marzo de I94I, pp. 3I3-32I.

I5. "Elogio de Chile" [Cambió el título por "Elogio del terruño" en Alma y cuerpo de Cbile], LXVI, diciembre de I94r, pp. 3r8-324.

I6. "Significación de Lastarria", LXVIII, mayo de I942, pp. 228-232.

17. "Algunos aspectos de la literatura peruana, hasta Ciro Ảlegría", LXX, diciembre de I942, pp. 278-308.

r8. "Casa de la infancia", LXXII, abril de I943; pp. 8-22.

I9. "Impresión galdosiana", LXXII, mayo de I943, Pp. I60-r64.

20. " "La sal, el pan y el vino" [Cambió el título por "Alma y cuerpo de Chile" en el libro del mismo nombre], LXXXI, diciembre de I945, pp. 3r8-332.

2r. "Adiós a Domingo [Melfi]", LXXXIII, marzo de r946, pp. 395398.

22. " "Escenario del huaso", LXXXVII, septiembre de I947, pp. 312-3I8.

23. "América y Cervantes", LXXXVIII, octubre de I947, Pp. 5-Io.

24. "El cuento chileno", XC, septiembre-octubre de I948, pp. I-7.

25. * "Afuerinos", XC, septiembre-octubre de I948, pp. 26r-274.

26. "Atenea' al servicio de la cultura", XCV, septiembre-octubre de I949, pp. 3-ro.

27. "Augusto d'Halmar", XCVI, enero-febrero de I950, Pp. 7-9.

28. "Concepción: el águila negra en campo de oro", XCIX, octubre de I950, Pp. 5-15.

29. "Augusto d'Halmar en el recuerdo", C, enero-febrero de I95I, Pp. I28-I33.

30. "En el asertadero", CV, enero-febrero de I952, pp. I9-23.

\section{SOBRE LUIS DURAND}

I. Acevedo, Olga, Crítica de Fronterd, Atenea, XCVI, enero-febrero de I950, Pp. I56-I58.

2. Alessandri Palma, Arturo, "Carta a Luis Durand", citada en Luis Durand, Sietecuentos (Santiago: Nascimento, I950).

3. Clariana, Abelardo, Los mejores cuentos y trozos selectos de Luis Durand (Santiago: Zig-Zag, r959), pp. 9-16. 
4. Díaz Arrieta, Hernán ["Alone"], Crítica de Frontera, citada en Luis Durand, Sietecuentos (Santiago: Nascimento, 1950).

5. Diccionario biográfico de Chile, Novena edición (Santiago: Empresa Periodística Chile, I955), p. 374.

6. Fuenzalida, Héctor, Crítica de El primer bijo, Atenea, XXXVII, enero de 1937, pp. 65-68.

- - "Luis Durand y sus novelas", en Cien años de la novela cbilena (Concepción: Ediciones Revista Atenea, r96r), pp. 90-ro2.

7. González Vera, José Santos, Prólogo a Luis Durand en Vino tinto, y otros cuentos (Santiago: Cruz del Sur, I943), pp. 7-ro.

8. Gutiérrez, Joaquín, "El gran linaje de la obra de Luis Durand", Atenea, CXVIII, noviembre-diciembre de 1954, pp. 6I-65.

9. Huerta, Eleazar, "El cuento chileno y Luis Durand", Atenea, XC, septiembre-octubre de 1948, pp. 27-33.

- "Recuerdo de Luis Durand", Akene'a, CXVIII, noviembrediciembre de I954, Pp. I2-I8.

ro. Jobet, Jorge, Crítica de Frontera, Atenea, XCVIII, agosto-septiembre de I950, Pp. 34r-344.

Ir. Lastra, Pedro, Crítica de Paisajes y gentes de Cbile, Atenea, CXVI, julio-agosto de 1954, Pp. I66-167.

I2. Latcham, Ricardo A., Prólogo a Luis Durand en Tierra de pellines (Santiago: Nascimento, I929), pp. 7-II.

I3. Latorre, Mariano, Prólogo a Luis Durand en La Chabela (Santiago: Lectura Selecta, r927), Pp. 3-4.

I4. Loveluck, Juan, "Notas sobre algunos cuentos de Luis Durand", Atenea, CXVIII, noviembre-diciembre de 1954, pp. 38-60.

15. Martínez, Carlos, "El premio 'Atenea' en la Universidad de Concepción", Atenea, XCIX, octubre de I950, Pp. I49-I53.

r6. Melfi, Domingo, "Un juicio sobre Luis Durand", en Campesinos, Segunda edición (Santiago: Nascimento, I950), pp. 3-Io.

17. Merino Reyes, Luis, Prólogo a Luis Durand en Guaugatu y sus amigos (Santiago: Rapa-Nui, I947), P. 9.

-, Prólogo a Luis Durand en Un amor (Santiago: Zig-Zag, 1958), Pp. 7-I7.

r8. Molina, Enrique, "Presencia de Luis Durand", Atenea, CXVIII, noviembre-diciembre de 1954, Pp. I-3.

—, Prólogo a Luis Durand en Presencia de Chile (Santiago: Nascimento, I942), Pp. I7-13. 
I9. Montaldo, Caupolicán, "Luis Durand, el amigo", Atenea, CXVIII, noviembre-diciembre de 1954 , pp. 66-68.

20. Montenegro, Ernesto, "La doble personalidad de Luis Durand", Atenea, CXVIII, noviembre-diciembre de I954, pp. I9-22.

2I. Núñez, Félix Armando, Crítica de Frontera, Atenea, C, enero-febrero de I95I, pp. I78-I80.

22. Osses, Mario, Crítica de Sietecuentos, Atenea, XCIX, noviembrediciembre de I950, pp. 368-372.

- "Sobre siete cuentos maestros de la literatura chilena", Atenea, $\mathrm{XC}$, septiembre-octubre de I948, pp. 34-62.

23. Perry B., David, Crítica de Piedra que rueda, Atenea, XXVII, octubre de I934, pp. 646-669.

- Crítica de Mercedes Urizar, Atenea, XXIX, enero de 1935. Pp. II4-II8.

24. Rojas, Gonzalo, "Luis Durand", Atenea, CXVIII, noviembre-diciembre de I954, PP. 4-II.

25. Romera, Antonio R., "El 'bodegón' en la obra de Luis Durand", Atenea, CXVIII, noviembre-diciembre de r954, pp. 23-32.

26. Rueda, Manuel, "Reflexiones acerca de Frontera y el criollismo", Aienea, XCVIII, julio de I950, pp. I39-I44.

27. Sánchez, Luis Alberto, "Para una antología de Luis Durand", Atenea, CXVIII, noviembre-diciembre de I954, PP. 69-106.

28. Silva Castro, Raúl, Diccionario de la literatura latinoamericana: Cbile (Washington: Unión Panamericana, I958), pp. 63-64.

29. Torres Ríoseco, Arturo, Prólogo a Luis Durand en Cielos del sur (Santiago: "Cultura", r933), pp. ix-xiv.

30. Uriarte, Fernando, "Realidad y posibilidad de Luis Durand", Ate" nea, CXVIII, noviembre-diciembre de I954, pp. 33-37.

3I. Yankas, Lautaro, "Esquema de Luis Durand", Atenea, XXVI, junio de $x 934$, pp. 516-520.

Donald M. Decker

University of the Pacific 
